\title{
Prognostic significance of thrombocytosis in patients with primary lung cancer
}

\author{
L. Møller Pedersen, N. Milman
}

Prognostic significance of thrombocytosis in patients with primary lung cancer. L. Mфller Pedersen, N. Milman. OERS Journals Ltd 1996.

ABSTRACT: In patients with malignancies, thrombocytosis has previously been related to disease stage, histological type, and survival. In the present study, the prevalence of thrombocytosis and the prognostic information provided by platelet counts were analysed in a large cohort of patients with primary lung cancer.

At the time of diagnosis, pretreatment platelet counts were retrospectively recorded in 1,115 consecutive patients with histologically proven primary lung cancer. All patients were reviewed regarding histological type, tumour, node, metastasis (TNM) classification stage and survival. The prevalence of thrombocytosis in patients with lung cancer was compared with that in a series of 550 consecutive out-patients with benign lung disorders. In 269 surgically resected patients, postoperative platelet counts were recorded 1-3 months after resection of the tumour. In the follow-up period, thromboembolic episodes diagnosed either clinically or at autopsy were recorded.

The overall prevalence of thrombocytosis $\left(>400 \times 10^{9}\right.$ platelets $\left.{ }^{-1}{ }^{-1}\right)$ in the patients with lung cancer was $32 \%$. The frequency of thrombocytosis was significantly higher compared with the control subjects $(32$ vs $6 \% ; \mathbf{p}<0.0001)$. Platelet counts differed significantly among subgroups defined by the TNM classification, with the proportion of patients with $>400 \times 10^{9}$ platelets $\mathrm{L}^{-1}$ greatest in the more advanced TNM stages (stage I and II $23 \%$ vs stage III and IV 37\%; p<0.0001). Patients with thrombocytosis had a significantly poorer survival than patients with normal platelet counts $(\mathbf{p}<0.0001)$. In a multivariate survival analysis $(\mathrm{Cox}$ model), thrombocytosis continued to correlate strongly with poor survival even when adjusted for histological type, sex, age, and TNM stage $(\mathrm{p}<0.001)$. In surgically resected patients, the frequency of preoperative and postoperative thrombocytosis differed significantly (23.0 vs $8.9 \% ; \mathbf{p}<0.0001$ ). Survival rate was significantly reduced in patients with preoperative thrombocytosis $(\mathrm{p}=\mathbf{0 . 0 0 5})$. Thrombocytosis was not associated with an increased incidence of thromboembolism.

In conclusion, thrombocytosis is an independent prognostic factor of survival in patients with primary lung cancer. We suggest that platelet counts should be included in future multivariate analyses of survival in patients with lung cancer.

Eur Respir J., 1996, 9, 1826-1830.
Dept of Pulmonary Medicine, Gentofte Hospital, University of Copenhagen, Denmark.

Correspondence: L. Møller Pedersen Dept of Medicine Roskilde Hospital, Køgevej 7-13

DK-4000 Roskilde

Denmark

Keywords: Lung cancer

platelet count

prognostic factors

thrombocytosis

tumour markers

Received: April 231996

Accepted after revision May 231996
The association between malignancy and elevated platelet counts has been recognized for almost a century [1]. Several studies have confirmed thrombocytosis as a paraneoplastic symptom [2-5]. Many patients presenting with thrombocytosis have an underlying malignant disease [2]. The pathogenesis of thrombocytosis in malignancy has not yet been clarified. However, there is evidence that the tumour cells secrete humoral factors which may eventually lead to thrombocytosis [6].

A number of factors have prognostic impact on patients with lung cancer but, apart from disease stage and performance status, such features are rarely considered to be of clinical value in the individual patient [7-9]. There is increasing evidence that an interaction between tumour cells, endothelial cells, and platelets may contribute to the development of metastases resulting in poorer prognosis [10]. However, the prognostic significance of platelet counts has not yet been adequately clarified [11, 12].

In this study, we report the prevalence of thrombocytosis in a large population of consecutive patients with primary lung cancer. The association between pretreatment platelet count, disease stage, incidence of thromboembolic episodes, and survival was analysed. Furthermore, the impact of surgical resection of the tumour on platelet counts was investigated.

\section{Materials and methods}

The present study comprises 1,178 consecutive patients with primary lung cancer, diagnosed at the Department of Pulmonary Medicine, Gentofte University Hospital, Copenhagen, between 1986 and 1993. Excluded from the study were subjects with concomitant myeloproliferative disorders or conditions known to be associated with reactive thrombocytosis, such as inflammatory diseases, autoimmune disorders and other malignancies $(n=29)$. Furthermore, patients without available platelet counts were excluded $(n=34)$. Medical records of the remaining 1,115 patients were retrospectively reviewed. They consisted of 822 
males and 293 females, with a median age of 58 yrs (range 28-91 yrs).

Each patient was staged according to the tumour, node, metastasis (TNM) system of classification [13]. Clinical staging included chest radiography, a computed tomographic (CT) scan of the thorax and, in some cases, of the upper abdomen and brain, fibreoptic bronchoscopy with endobronchial or transbronchial biopsies and bronchial washing, cytological examination of sputum and pleural fluid, percutaneous transthoracic needle biopsy and/or mediastinoscopy. When necessary, explorative thoracotomy was performed. In addition, a bone scan and a bone marrow biopsy were performed in patients with small cell lung cancer. The histopathological tumour diagnoses were obtained from the local Department of Pathology and classified according to the World Health Organisation (WHO) [14].

In 309 surgically resected patients with non-small cell lung cancer, postoperative platelet counts were available in $269(87 \%)$ patients. Only those platelet counts taken 1-3 months after thoracotomy were recorded because of the possibility of reactive thrombocytosis induced by the surgical procedure. One hundred and ninety one (62\%) patients were considered to be radically resected. Resection was defined to be radical when the primary tumour was excised along with all positive hilar and mediastinal lymph nodes and with histologically proven negative margins. Five $(1.6 \%)$ patients dying of complications directly associated with the surgical procedure before discharge from the hospital (operative deaths) were not included. Platelet counts following chemotherapy or radiation therapy were not recorded in this study due to the possible effects of treatment on platelet levels.

The prevalence of thrombocytosis was compared with 550 consecutive out-patients with benign lung disorders, admitted to our clinic in the same period of time as the patients with malignancies. In accordance with the lung cancer patients, control subjects with disorders considered to be associated with reactive thrombocytosis were excluded. The age distribution did not differ significantly between patients with lung cancer and control subjects (median age 58 vs 56 yrs). The male:female ratio in patients with lung cancer and control subjects was 2.8 and 1.9 , respectively.

Thromboembolic events in the follow-up period were recorded in each patient. Episodes of thromboembolism were diagnosed either clinically or at autopsy. Few patients came to postmortem examination (autopsy rate 14\%), with no significant difference in frequency of autopsy between patients with and without thrombocytosis (16 vs 13\%). Deep venous thrombosis was diagnosed by venogram and pulmonary embolism by pulmonary perfusion-ventilation technetium scanning or at autopsy. Coronary thrombosis was diagnosed clinically by electrocardiographic changes and serum enzyme elevation, or at autopsy. Cerebral thromboembolism was diagnosed by cerebral CT or magnetic resonance (MR) scanning, or by physical examination. Femoral artery thrombosis was diagnosed by angiography. Disseminated intravascular coagulation and renal venous thrombosis were both diagnosed at autopsy.

The pretreatment platelet counts used were obtained at the initial visit to the clinic before invasive diagnostic procedures were performed. Platelet counts were analysed by automated complete blood cell counting devices on ethylenediamine tetra-acetic acid (EDTA)-anticoagulated blood. In our laboratory, the $95 \%$ confidence limit (normal range) of platelet count is defined as 150-400 $\times 10^{9} \cdot \mathrm{L}^{-1}$. The platelet count was considered as a dichotomous variable. In agreement with other studies, thrombocytosis was defined as a platelet count of $>400 \times 10^{9} \cdot \mathrm{L}^{-1}$.

Data are expressed as median values. The Mann-Whitney U-test was used to assess group differences in continuous variables. Group differences in categorical variables were analysed by the Chi-squared test. Survival time was calculated from the date of the histologically confirmed diagnosis to death or to the day of follow-up (May 1995). At the time of follow-up, $980(88 \%)$ patients had died. Median time from study entry to the date of follow-up for the 135 (12\%) living patients was 52 months (range 16-109 months). Univariate survival analyses were calculated using the Kaplan-Meier life tables [15]. Differences between survival curves were tested by the log-rank test [16]. The relative importance of multiple factors on survival was estimated using Cox's proportional hazards regression model [17]. A p-value of $<0.05$ was considered significant. Statistical analysis was performed by means of the BMDP software package [18].

\section{Results}

The overall distribution of platelet counts in lung cancer patients and controls is shown in figure 1. Patients with lung cancer had a significantly higher prevalence of thrombocytosis than the control subjects $(358 / 1115$ $(32 \%)$ vs $35 / 550(6 \%) ; \mathrm{p}<0.0001)$. No significant age difference was apparent when cancer patients with thrombocytosis were compared to patients with normal platelet counts (median age 60 vs 57 yrs). Stage and histological type distributions in the study population are listed in table 1. Pretreatment thrombocytosis was more common in patients with advanced disease (table 2). The frequency of thrombocytosis was $23 \%$ in patients with TNM stage I and II and increased to $37 \%$ in patients with TNM stage III and IV. This difference in platelet count distribution according to disease stage was statistically significant $(p<0.0001)$. The difference was further increased, if the frequency of platelet counts $>600 \times 10^{9} \cdot \mathrm{L}^{-1}$ in patients with limited and advanced disease was compared (9\% vs 22\%;

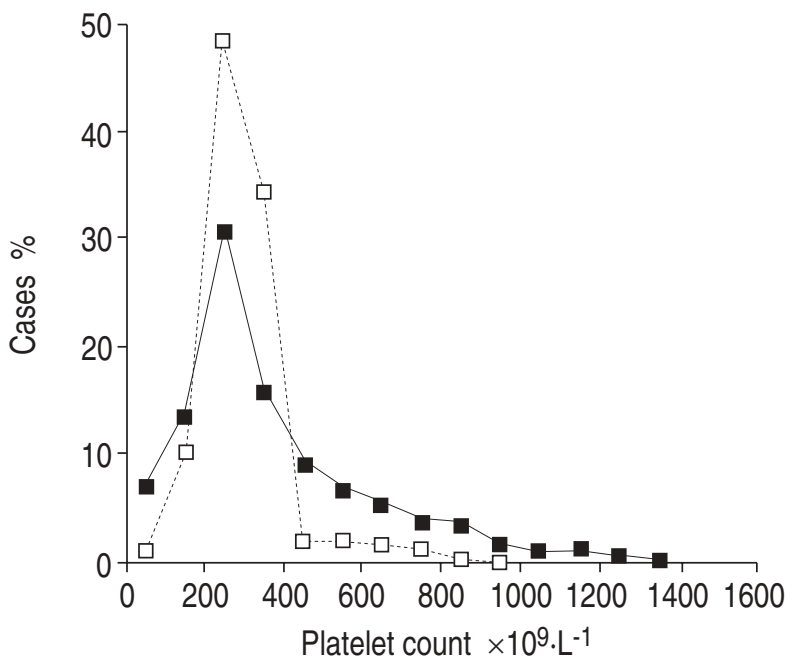

Fig. 1. - Distribution of platelet counts $\left(\times 10^{9} \cdot \mathrm{L}^{-1}\right)$ in 1,115 patients with primary lung cancer (- $\square$ ) and in 550 control subjects $(\cdots \square \cdots \cdots)$. 
Table 1. - Study population of patients with primary lung cancer described by histological type and disease stage at presentation

\begin{tabular}{lcc}
\hline & $\mathrm{n}$ & Patients \\
\hline Histological diagnosis & & $\%$ \\
Squamous cell carcinoma & 435 & 39 \\
Adenocarcinoma & 284 & 25 \\
Large cell carcinoma & 153 & 14 \\
Small cell carcinoma & 243 & 22 \\
TNM stage & & \\
I & 107 & 10 \\
II & 286 & 26 \\
IIIa & 283 & 25 \\
IIIb & 307 & 27 \\
IV & 132 & 12 \\
\hline
\end{tabular}

TNM: tumour, node, metastasis classification.

Table 2. - Platelet counts at diagnosis in patients with primary lung cancer according to TNM stages

\begin{tabular}{lrrcc}
\hline TNM stage & \multicolumn{4}{c}{ Cases $>400$} \\
& $\mathrm{n}$ & $\%$ & Median value & $\begin{array}{l}\text { 25th-75th } \\
\text { percentile }\end{array}$ \\
\hline I & $21 / 107$ & 20 & 240 & $157-341$ \\
II & $71 / 286$ & 25 & 262 & $166-341$ \\
IIIa & $91 / 283$ & 32 & 317 & $223-488$ \\
IIIb & $121 / 307$ & 39 & 328 & $248-534$ \\
IV & $54 / 132$ & 41 & 351 & $283-670$ \\
\hline
\end{tabular}

TNM: tumour, node, metastasis classification.

$\mathrm{p}<0.0001)$. There was no significant difference in the distribution of platelet counts between the histological types.

Preoperative platelet counts were above $400 \times 10^{9} \cdot \mathrm{L}^{-1}$ in $62(23 \%)$ of the surgically resected patients, whereas postoperative thrombocytosis occurred in only $24(9 \%)(\mathrm{p}<$ $0.0001)$. However, the difference in frequency of postoperative thrombocytosis between patients with radical surgery and patients with postoperative residual disease did not reach statistical significance (8vs 10\%).

Episodes of thromboembolism in patients with and without thrombocytosis are shown in table 3 . The frequency of thromboembolism in patients with thrombocytosis was not statistically different from the frequency found in

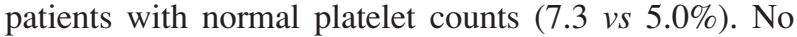
fatal thromboembolic events were observed in the follow-up period.

In the univariate survival analysis of the 1,115 patients with primary lung cancer, the overall survival rate was $47 \%$ after $1 \mathrm{yr}, 23 \%$ after 2 yrs, and $14 \%$ after 5 yrs. The total 5 year survival was higher than expected but is explained by patient selection in a specialized hospital which cannot be truly representative of the entire population. Patients considered to be unfit for active treatment may not have been referred. Survival curves for patients with thrombocytosis and patients with normal platelet counts are shown in figure 2. Patients with elevated platelet counts had a significantly poorer survival rate than those with normal platelet counts $(\mathrm{p}<0.0001)$. The probability of survival at 1, 2 and $5 \mathrm{yrs}$ after diagnosis was 38, 13 and $4 \%$ vs 51, 28 and $18 \%$, respectively. If $600 \times 10^{9} \cdot \mathrm{L}^{-1}$ was selected as the cut-off value for platelet counts, the difference between the two survival curves was further increased $(\mathrm{p}<0.0001)$. In surgically resected patients, the survival rate was significantly lower in patients with pretreatment thrombocytosis $(\mathrm{p}=0.005)$. Post-operative thrombocytosis had no significant influence on survival.
Table 3. - Number of thromboembolic episodes in patients having primary lung cancer with $(n=358)$ and without $(n=757)$ thrombocytosis

\begin{tabular}{lrc}
\hline & \multicolumn{2}{c}{ Platelet count } \\
\cline { 2 - 3 } Thromboembolic episode & $>400$ & $\leq 400$ \\
& $\times 10^{9} \cdot \mathrm{L}^{-1}$ & $\times 10^{9} \cdot \mathrm{L}^{-1}$ \\
\hline Deep venous thrombosis & 10 & 16 \\
Pulmonary embolism & 5 & 6 \\
Disseminated intravascular coagulation & 3 & 3 \\
Coronary thrombosis & 2 & 5 \\
Cerebral thromboembolism & 4 & 5 \\
Femoral artery thrombosis & 2 & 1 \\
Superior mesenteric venous thrombosis & 0 & 1 \\
Renal venous thrombosis & 0 & 1 \\
\hline Total & 25 & 38 \\
\hline
\end{tabular}

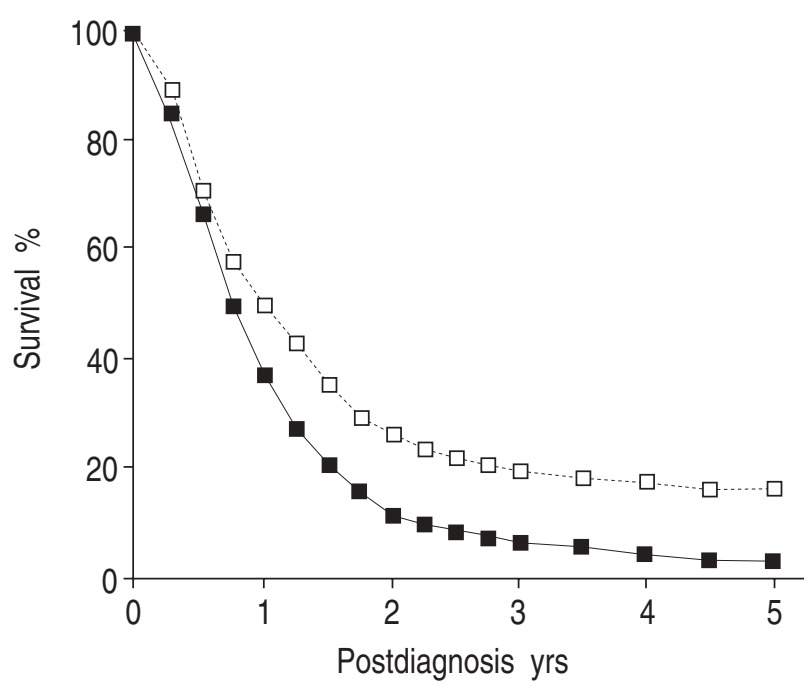

Fig. 2. - Survival of lung cancer patients stratified to normal $(\cdots \square \cdots \cdots)$ and elevated $(\longrightarrow-)$ platelet count.

Table 4. - Results of multivariate survival analysis (Cox model) with each variable adjusted by the others

\begin{tabular}{lccr}
\hline Variable & $\begin{array}{c}\text { Relative risk } \\
\text { estimates }\end{array}$ & $95 \%$ CI & p-value \\
\hline $\begin{array}{l}\text { Platelet count } \\
\leq 400 \times 10^{9} \cdot \mathrm{L}^{-1}\end{array}$ & 1 & & \\
$>400 \times 10^{9} \cdot \mathrm{L}^{-1}$ & 4.24 & $1.50-12.72$ & $<0.001$ \\
TNM stage & 1 & & \\
I & 2.45 & $1.26-4.64$ & 0.005 \\
II & 4.12 & $1.92-9.15$ & $<0.001$ \\
IIII & 4.94 & $1.76-13.74$ & $<0.001$ \\
IIIb & 5.33 & $1.78-15.07$ & $<0.001$ \\
IV & & & \\
Histological type & 1 & & \\
Squamous cell carcinoma & 1.24 & $0.53-2.27$ & NS \\
Adenocarcinoma & 1.67 & $0.66-2.89$ & NS \\
Large cell carcinoma & 1.92 & $0.74-4.42$ & NS \\
Small cell carcinoma & & & \\
Age & 1 & & \\
$<50$ yrs & 1.12 & $0.77-2.35$ & NS \\
50-70 yrs & 1.95 & $0.62-4.68$ & NS \\
$>70$ yrs & 1 & & \\
Sex & 1.63 & $0.84-3.04$ & NS \\
Male & Female & &
\end{tabular}

TNM: tumour, node, metastasis classification; $95 \%$ CI: $95 \%$ confidence interval; NS: nonsignificant. 
In the multivariate model, stepwise proportional hazards regression analysis of histological type, sex, age, and disease stage was performed (table 4 ). When adjusted for these factors, elevated platelet count continued to be a strong predictor of survival $(p<0.001)$. Among the other factors, only TNM staging had a significant role in the multivariate model. Histopathology, age, and sex did not exhibit statistical significance.

\section{Discussion}

Reactive thrombocytosis is commonly observed in neoplastic diseases [19-21]. The purpose of this study was to examine data on platelet counts obtained in a large population of patients with primary lung cancer to provide information on the prevalence of thrombocytosis and the association to survival. The frequency of elevated platelet counts in patients with primary lung cancer was significantly higher compared to patients with benign pulmonary diseases. In accordance with other reports, we found thrombocytosis in one third of patients with primary lung cancer. However, the frequencies of thrombocytosis reported in other studies have varied between 10 and 60\% [22-25]. Difficulties in interpreting abnormalities of the platelet count in malignancy arise because several conditions may influence the platelet levels. Malignancy is often accompanied by disseminated intravascular coagulation and bone marrow involvement, which tend to lower peripheral platelet counts. Treatment with chemotherapy and radiation therapy also diminish platelet numbers. The results of some reports may be debatable on account of small sample sizes or because platelet counts may have been influenced by diagnostic or therapeutic procedures. Furthermore, the studies may have dealt with different cohorts of patients with differences in stage distribution. In the present study, considering platelet counts influenced only by malignancy in a large population, a pronounced frequency of thrombocytosis was demonstrated. The distribution of platelet counts did not differ in the histological types in these patients. Other studies have reported higher platelet counts in non-small cell lung carcinoma [22].

High circulating levels of platelets are found in patients with metastatic disease and have been related to extensive disease in patients with lung cancer and other malignancies $[3,23,24,26]$. The present study confirms that thrombocytosis is related to extended tumour spread, as shown by the association of the prevalence of elevated platelet counts with disease stage reflecting the actual tumour load. Moreover, a significant difference was disclosed between preoperative platelet counts and platelet counts after surgical resection of the tumour mass. The results suggest that thrombocytosis is a marker of advanced disease and may be the result of substances produced by the larger tumour load. In a few other reports, thrombocytosis has remitted following definitive radiotherapy, chemotherapy or surgical resection of the primary lesion [27].

Elevated levels of platelet counts have been found to be a significant prognostic factor in carcinoma of the lung $[12,28]$. However, the association between thrombocytosis and survival has been questionable in multivariate analyses $[8,11]$. Thrombocytosis has also been revealed as an indicator of poor prognosis in patients with other malignancies [19, 29]. In other studies, platelet levels did not have a significant effect on patient survival [28]. Thrombocytosis as a valuable prognostic marker is strongly supported by the present results. These data prove the prognostic ability of platelet counts in lung cancer and the independence of this information from that provided by the other factors included. In a multivariate survival analysis, thrombocytosis continued to be a highly significant predictor of short-term survival when adjusted for other prognostic factors including TNM stage. However, the estimation of the relative importance of platelet count on survival would have been more valid if performance status was included in the multivariate model. Reliable information about the performance score could not be obtained in this retrospective study. Thrombocytosis may be of prognostic value due to interactions between the platelets and the cancer cells. Several lines of evidence provide strong support for the concept that tumour cell interaction with platelets contributes to haematogenous metastases [30]. Platelet-secreted proteins have been shown to potentiate tumour cell metastases [31].

Haemostatic abnormalities are frequently observed in patients with malignancy [32]. Most patients with cancer have evidence of subclinical activation of blood coagulation [33]. Clinically, cancer patients with advanced disease are characterized by a variety of thromboembolic disorders. The pathophysiological mechanisms inducing hypercoagulability in cancer patients are complex. However, reactive thrombocytosis in malignant diseases has not been regarded as a clinically important factor because of the rarity of vaso-occlusive complications [34]. Statistical analysis of our series supports this view because the frequency of thromboembolic events was not correlated to the presence of thrombocytosis. The incidence of thromboembolic episodes in patients with elevated and normal platelet counts was 7 and 5\%, respectively. The autopsy rate in these patients was rather low and the occurrence of thromboembolic events may have been underestimated. The frequency of thrombosis reported in other studies is typically $5-10 \%[35,36]$. No fatal thromboembolic episodes occurred in the present study. Serious thrombotic events in patients with reactive thrombocytosis have only been described in a few case reports $[37,38]$. Thus, thromboembolic disease in cancer patients seems to be associated with other abnormalities of blood coagulation [33].

Neither the underlying explanation for the predictive value of platelet count nor the relationship between platelet expression and biological behaviour of cancer cells is clarified. The pathophysiological mechanism of reactive thrombocytosis may be tumour-associated elevations of circulating platelets mediated by a direct promotion of megakaryocytopoiesis by tumour-derived humoral factors. Interleukin-6 (IL-6) and macrophage colony-stimulating factor (M-CSF) may be responsible for the development of cancer-related thrombocytosis $[39,40]$. IL-6 is a potent stimulator of megakaryocytopoiesis and tumour cells have been shown to release IL-6 both in vitro and in vivo $[41,42]$. These observations strongly suggest a role for IL-6 in tumour-associated thrombocytosis.

In conclusion, we report a high prevalence of elevated platelet counts in patients with lung cancer. Thrombocytosis should not be considered as a risk factor predisposing to thromboembolism in cancer patients. Thrombocytosis was associated with the tumour load according to disease stage. Surgical debulking of the tumour resulted in significantly 
reduced platelet counts. Thrombocytosis displayed a highly significant relationship to survival. We suggest that platelet counts are included in future multifactorial analyses of survival in patients with lung cancer.

\section{References}

1. Riess L. Zur pathologischen Anatomie des Blutes. Arch Anat Physiol Wissensch Med 1872; 39: 237-249.

2. Levin J, Conley CL. Thrombocytosis associated with malignant disease. Arch Intern Med 1964; 114: 497-500.

3. Edwards RL, Rickles FR, Moritz TE, et al. Abnormalities of blood coagulation tests in patients with cancer. Am J Clin Pathol 1987; 88: 596-602.

4. Dutcher JA. Hematologic abnormalities in patients with nonhematologic malignancies. Hematol Oncol Clin N Am 1987; 1: 281-299.

5. Nand S, Gross-Fisher S, Salgia R, Fisher RI. Hemostatic abnormalities in untreated cancer: incidence and correlation with thrombotic and hemorrhagic complications. J Clin Oncol 1987; 5: 1998-2003.

6. Ascensao JL, Oken MM, Ewing SL, Goldberg RJ, Kaplan ME. Leukocytosis and large cell lung cancer. Cancer 1987; 60: 903-905.

7. Souhami RL, Bradbury J, Geddes DM, Spiro SG, Harper PG, Tobias JS. Prognostic significance of laboratory parameters measured at prognosis in small cell carcinoma of the lung. Cancer Res 1985; 45: 2878-2882.

8. Østerlind K, Andersen PK. Prognostic factors in small cell lung cancer: multivariate model based on 778 patients treated with chemotherapy with or without irradiation. Cancer Res 1986; 46: 4189-4194.

9. Cohen MH, Makuch R, Johnston-Early A, et al. Laboratory parameters as an alternative to performance status in prognostic stratification of patients with small lung cancer. Cancer Treat Rev 1981; 65: 187-195.

10. Warrell RP Jr, Bockman RS, Staszewski H, Maiese K. Clinical study of a new antimetastatic compound, nafazatrom (Bay g 6575): effects on platelet consumption and monocyte prostaglandin production in patients with advanced cancer. Cancer 1986; 57: 1455-1460.

11. Gislason T, Nou E. Sedimentation rate, leucocytes, platelet count and haemoglobin in bronchial carcinoma: an epidemiological study. Eur J Respir Dis 1985; 66: 141-146.

12. Engan T, Hannisdal E. Blood analyses as prognostic factors in primary lung cancer. Acta Oncologica 1990; 29: $151-154$.

13. Mountain CF. A new international staging system for lung cancer. Chest 1986; 89: 225-233.

14. Histological typing of lung tumours. 2nd edn. Geneva, World Health Organization, 1981.

15. Kaplan EL, Meier P. Nonparametric estimation from incomplete observations. J Am Stat Assoc 1958; 53: 457-481.

16. Peto R, Pike MC, Armitage R, et al. Design and analysis of clinical trials requiring prolonged observation of each patient. Br J Cancer 1977; 35: 1-47.

17. Cox DR. Regression model and life tables. J R Stat Soc (Series B) 1972; 34: 187-220.

18. BMDP statistical software. Berkeley, CA, University of California Press, 1981

19. Hernandez E, Lavine M, Dunton CJ, Gracely E, Parker J. Poor prognosis associated with thrombocytosis in patients with cervical cancer. Cancer 1992; 69: 2975-2977.

20. Zeimet AG, Marth C, Müller-Holzner E, Daxenbichler G, Dapunt O. Significance of thrombocytosis in patients with epithelial ovarian cancer. Am J Obstet Gynecol 1994; 170: 549-554.

21. Chalas E, Welshinger M, Engellener W, Chumas J,
Barbieri R, Mann WJ. The clinical significance of thrombocytosis in women presenting with a pelvic mass. Am J Obstet Gynecol 1992; 166: 974-977.

22. Constantini V, Zacharski LR, Moritz TE, Edwards RL. The platelet count in carcinoma of the lung and colon. Thromb Haemost 1990; 64: 501-505.

23. Silvis SE, Turkbas N, Doscherholmen A. Thrombocytosis in patients with lung cancer. JAMA 1979; 211: 1852-1853.

24. Diehl WL, Mandelbaum I. The significance of thrombocytosis in patients with carcinoma of the lung. Surg Gynecol Obstet 1983; 156: 187-188.

25. Nakano T, Fujii J, Tamura S, Hada T, Higashino K. Thrombocytosis in patients with malignant pleural mesothelioma. Cancer 1986; 58: 1699-1701.

26. Wajima T, Mukhopadhyay P. Serial coagulation profiles in patients with small cell carcinoma of the lung (Abstract). Thromb Haemost 1989; Suppl. 62: 136.

27. Spigel SC, Mooney LR. Extreme thrombocytosis associated with malignancy. Cancer 1977; 39: 339-341.

28. Lopes A, Daras V, Cross PA, Robertson G, Beynon G, Monaghan JM. Thrombocytosis as a prognostic factor in women with cervical cancer. Cancer 1994; 74: 90-92.

29. Rodriguez GC, Clarke-Pearson DL, Soper JT, Berchuck A, Synan I, Dodge RK. The negative prognostic implications of thrombocytosis in women with stage Ib cervical cancer. Obstet Gynecol 1994; 83: 445-448.

30. Honn KV, Tang DG, Crissman JD. Platelets and cancer metastasis: a causal relationship? Cancer Metastasis Rev 1992; 11: 325-351.

31. Tuszynski GP, Gasic TB, Rothman VL, Knudsen KA, Gasic GJ. Thrombospondin: a potentiator of tumor cell metastasis. Cancer Res 1978; 47: 4130-4133.

32. Sun NC, McAfee WM, Hum GJ, Weiner JM. Hemostatic abnormalities in malignancy, a prospective study of one hundred and eight patients. Part 1. Coagulation studies. Am J Clin Pathol 1979; 71: 10-16.

33. Rickles FR, Levine M, Edwards RL. Hemostatic alterations in cancer patients. Cancer Metastasis Rev 1992; 11: 237-248.

34. Buss DH, Cashell AW, O'Connor ML, Richards F, Case LD. Occurrence, etiology, and clinical significance of extreme thrombocytosis: a study of 280 cases. Am J Med 1994; 96: 247-253.

35. Lieberman JS, Borrero J, Urdaneta E, Wright IS. Thromboembolism associated with neoplasm: review of seventy seven cases. JAMA 1961; 177: 542-545.

36. Miller SP, Sanchez-Avalos J, Stefanski T, Zuckerman L. Coagulation disorders in cancer. I. Clinical and laboratory studies. Cancer 1967; 20: 1452-1456.

37. Alexander MB. Iron deficiency anemia, thrombocytosis, and cerebrovascular accident. South Med J 1983; 76: 662-663.

38. Knizley H, Noyes WD. Iron deficiency anemia, papilledema, thrombocytosis and transient hemiparesis. Arch Intern Med 1972; 129: 483-486.

39. Imai T, Koike K, Kubo T, et al. Interleukin-6 supports human megakaryocyte proliferation and differentiation in vitro. Blood 1991; 78: 1969-1964.

40. Ramakrishnan S, Xu FJ, Brandt SJ, Niedel JE, Bast RC $\mathrm{Jr}$, Brown EL. Constitutive production of macrophage colony-stimulating factor by human ovarian and breast cancer cell lines. J Clin Invest 1989; 83: 921-926.

41. Gastl G, Plante M, Finstad CL, et al. High IL-6 levels in ascitic fluid correlate with reactive thrombocytosis in patients with epithelial ovarian cancer. Br J Haematol 1993; 83: 433-441.

42. Hollen CW, Henthorn J, Koziol JA, Burstein SA. Elevated serum interleukin-6 levels in patients with reactive thrombocytosis. Br J Haematol 1991; 79: 286-290. 